\title{
Transplante de córnea em Alagoas: aspectos clínicos e epidemiológicos do Hospital Universitário Professor Alberto Antunes
}

\author{
Corneal transplantation in the Alagoas State: clinical and epidemiological aspects \\ of a reference service at Hospital Universitário Professor Alberto Antunes
}

Renata Leonel Freire Mendes ${ }^{1}$ (), Andrea Maria Cavalcante Santos ${ }^{2}$ (D), Alex Mendes Leonel Freire ${ }^{3}$ (])

1 Programa de Fellowship em Catarata, Clínica Oftalmológica do Hospital das Clínicas da Faculdade de Medicina da Universidade de São Paulo, São Paulo, SP, Brasil 2 Departamento de Oftalmologia, Hospital Universitário Professor Alberto Antunes, Universidade Federal de Alagoas, Maceió, AL, Brasil. ${ }^{3}$ Programa de Residência em Oftalmologia, Universidade Estadual Paulista "Júlio de Mesquita Filho", Botucatu, SP, Brasil.

Transplante de córnea;

Ceratoplastia penetrante;

Doenças da córnea/diagnóstico

Keywords:

Corneal transplantation;

Keratoplasty, penetrating; Corneal diseases/diagnosis

Recebido: $8 / 4 / 2020$

Aceito:

21/2/2021

Autor correspondente: Renata Leonel Freire Mendes Avenida Sandoval Arroxelas, 112/303-a Ponta Verde CEP: 57035-230 - Maceió, AL, Brasil E-mail: renataleonelfm@gmail.com

Instituição: Departamento de Oftalmologia, Hospital Universitário Professor Alberto Antunes, Universidade Federal de Alagoas, Maceió, AL, Brasil.

Fonte de auxílio à pesquisa: não financiado.

Conflitos de interesse: os autores declaram que não há conflitos de interesses.

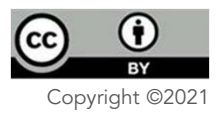

\section{RESUMO}

Objetivo: Descrever o perfil clínico, cirúrgico e geográfico de pacientes acompanhados em um hospital universitário e submetidos a transplante de tecido corneano penetrante, com descrição das indicações para o procedimento e do tempo médio na fila de espera.

Métodos: Estudo transversal e retrospectivo, incluindo 40 olhos de 40 pacientes submetidos à ceratoplastia penetrante no período de $1^{\circ}$ de janeiro de 2018 a 31 de dezembro de 2019, acompanhados em um hospital universitário em Alagoas. Os dados foram coletados por meio das fichas de informações cirúrgicas dos transplantes de córnea da Central de Notificação, Captação e Distribuição de Órgãos e Tecidos de Alagoas, adaptados aos objetivos da pesquisa.

Resultados: Dos transplantes de córnea estudados, 52,5\% ( $n=21)$ foram realizados em pacientes do sexo feminino, 62,5\% ( $n=25)$ na faixa etária acima de 60 anos, com média de idade de 59,17 anos $( \pm 20,4)$. Todos ocorreram em pacientes que residiam no estado de Alagoas, sendo $60 \%$ deles na região intermediária de Maceió. As principais indicações para o transplante de córnea foram ceratopatia bolhosa do pseudofácico ( $n=11 ; 27,5 \%)$, descemetocele $(n=9 ; 22,5 \%)$ e falência tardia do enxerto ( $n=6$; $15 \%)$. Dentre os procedimentos, $70 \%$ foram realizados no olho esquerdo $(n=28)$ e $65 \%(n=26)$ com propósito óptico; houve associação de extração de catarata em $22,5 \%(n=9)$, e $5 \%(n=2)$ apresentaram complicações peroperatórias. O tempo médio geral em lista de espera foi de 332,3 dias (11 meses), sendo 486 dias (2 anos e 4 meses) para cirurgias eletivas e 12,8 dias para as de urgência.

Conclusão: $\bigcirc$ tempo de espera para cirurgias eletivas foi longo e inadequado. A principal condição indicadora para o transplante de córnea foi a ceratopatia bolhosa. O conhecimento do perfil dos transplantes de córnea pode permitir a identificação de grupos de risco para fins de prevenção e implementação de cuidados, que resultem em prognósticos mais favoráveis, bem como incentivar a implementação de políticas internas e externas para melhoria do sistema captação-doação.

\section{ABSTRACT}

Objective: To describe the clinical, surgical, and geographic profile of patients submitted to corneal transplantation and followed up at a teaching hospital, and, with a description of the indications for the procedure and mean waiting time.

Methods: A cross-sectional and retrospective study, including 40 eyes of 40 patients who underwent penetrating keratoplasty, from January 1, 2018 to December 31, 2019, followed up at a teaching hospital in Alagoas State. Data were collected using the surgical records of corneal transplants, from the Central Reporting, Procurement and Distribution of Organs and Tissues of Alagoas, and adjusted to the research objectives.

Results: Of the corneal transplants studied, $52.5 \%(n=21)$ were performed in female patients; $62.5 \%$ $(n=25)$ in the age group over 60 years; with a mean age of 59.17 years $( \pm 20.4)$. All transplanted patients lived in the state of Alagoas, $60 \%$ of them in the intermediate metropolitan region of Maceió. The main indications for corneal transplantation were pseudophakic bullous keratopathy $(27.5 \%, n=11)$, descemetocele $(22.5 \%, n=9)$ and late graft failure $(15 \%, n=6)$. Seventy percent $(n=28)$ of procedures were performed on the left eye and $65 \%(n=26)$ for optical purposes. There was an association of cataract extraction in $22.5 \%(n=9)$, and $5 \%(n=2)$ had perioperative complications. The mean waiting list time was 332.3 days (11 months); in that, 486 days ( 2 years and 4 months) for elective surgeries and 12.8 days for emergency surgeries.

Conclusion: The waiting time for elective surgeries was long and inappropriate. The major indication for corneal transplantation was bullous keratopathy. Knowledge of the clinical profile of corneal transplants can enable identifying the risk groups for prevention and implementation of care, resulting in better prognosis, fostering implementation of internal and external policies to improve the procurementdonation system. 


\section{INTRODUÇÃO}

As doenças da córnea representam cerca de 4\% a 5\% das causas de cegueira reversível no mundo. ${ }^{(1,2)}$ Tais doenças apresentam várias etiologias, que incluem doenças crônicas, degenerativas, inflamatórias, infecciosas, distrofias e traumas. ${ }^{(3,4)}$ Se não forem adequadamente tratadas, essas doenças podem culminar em significativo impacto social nas pessoas acometidas, além de implicâncias psicológicas, que incluem isolamento social, depressão, ocorrência de acidentes, dependência familiar e até suicídio. ${ }^{(1,5)}$

O transplante de córnea, um efetivo tratamento para várias doenças graves de córnea, tem o objetivo de restaurar a transparência corneal. ${ }^{(6)}$ É o tipo de transplante mais frequente do mundo e tem se desenvolvido rapidamente nos últimos anos, com o desenvolvimento de novas técnicas cirúrgicas. ${ }^{(6)}$

Estudos realizados com o intuito de traçar o perfil dos pacientes que receberam o transplante de córnea, assim como daqueles em fila para o procedimento, em diversos espaços demográficos, permitem a comparação e a análise das variáveis determinantes para os mais diversos desfechos que envolvem o transplante corneano. Assim, o presente estudo se justifica diante da necessidade de determinar como o transplante de córnea varia de acordo com determinadas características clínicas que possibilitam a identificação de grupos de risco para fins de prevenção, além de gerar hipóteses para investigações posteriores. Espera-se que os resultados deste estudo possam contribuir para a melhoria da organização e o planejamento no atendimento aos usuários transplantados, bem como fomentar, na formação dos profissionais de saúde, a abordagem do assunto, de forma a propor cuidados especializados nos períodos pré e pós-operatórios pautados na detecção, na atenção e na prevenção das complicações.

O objetivo deste estudo foi descrever o perfil clínico, cirúrgico e geográfico dos pacientes acompanhados em serviço de referência e submetidos a transplante de tecido corneano, com descrição das indicações para o procedimento e do tempo médio na fila de espera.

\section{MÉTODOS}

O estudo realizado foi descritivo, transversal e retrospectivo, incluindo 40 olhos de 40 pacientes do total de 43 submetidos à ceratoplastia penetrante, única modalidade de transplante de córnea disponível no serviço, no período de $1^{\circ}$ de janeiro de 2018 a 31 de dezembro de 2019, acompanhados no Hospital Universitário Professor Alberto Antunes (HUPAA), da Universidade Federal de Alagoas (UFAL).
Os critérios de elegibilidade foram: indivíduos de todas as idades e de ambos os sexos e acompanhados pelo serviço dentro do período estudado, independentemente da condição clínica indicadora para realização do procedimento. Os dados foram coletados por meio das fichas de informações cirúrgicas dos transplantes de córnea da Central de Notificação, Captação e Distribuição de Órgãos e Tecidos (CNCDO) de Alagoas. Posteriormente, os registros foram analisados usando-se um roteiro estruturado, construído especificamente para sistematizar a coleta de dados.

Foram excluídos da pesquisa os pacientes que apresentaram dados incompletos na ficha de cadastramento, que corresponderam a três dos 43 pacientes submetidos ao procedimento.

Dentre as variáveis pesquisadas, estavam sexo, idade, cidade de origem, diagnóstico ocular, data de inclusão na CNCDO, data de realização do transplante, tempo decorrido na fila de espera até o transplante, ocorrência de complicações transoperatórias, combinação com extração de catarata e prioridade do transplante realizado.

Os dados foram processados, analisados pelo software Statistical Package for the Social Sciences (SPSS), versão 20.0, e apresentados em tabelas. Utilizou-se a estatística descritiva para análise univariada por meio de frequências absolutas e relativas, médias e medianas.

O protocolo de pesquisa foi aprovado pelo Comitê de Ética em Pesquisa da Universidade Federal de Alagoas em seus aspectos éticos e metodológicos, de acordo com a resolução 466/2012 do Conselho Nacional de Saúde.

\section{RESULTADOS}

Das 40 ceratoplastias penetrantes do presente estudo, $52,5 \%(n=21)$ foram realizadas em pacientes do sexo feminino. Com relação à idade, foram encontradas as seguintes frequências por intervalos de faixas etárias: até 20 anos, $7,5 \%(\mathrm{n}=3)$; de 21 a 30 anos, 7,5\% (n=3); de 31 a 40 anos, $2,5 \%(\mathrm{n}=1)$; de 41 a 50 anos, $10 \%(\mathrm{n}=4)$; de 51 a 60 anos, $10 \%(n=4)$ e acima de 60 anos, 62,5\% (n=25) (Tabela 1). A média da idade dos pacientes foi de 59,17 anos, com desvio-padrão de 20,4 anos, enquanto metade dos pacientes possuía idade de até 67,5 anos.

A figura 1 apresenta a distribuição geográfica dos casos de transplantes de córnea por regiões intermediárias (duas, demarcadas por linha espessa) e imediatas (11, demarcadas por linhas finas) do estado de Alagoas. Todos os 40 transplantes de córnea ocorreram em pacientes que residiam no próprio estado. Após o dimensionamento dos casos por local de residência do paciente, $60 \%(n=24)$ 
Tabela 1. Sexo e faixa etária dos pacientes submetidos a transplante de córnea ( $n=40$ olhos, 40 pacientes), de 2018 a 2019

\begin{tabular}{lc} 
Características & $\mathbf{n}(\%)$ \\
\hline Sexo & \\
$\quad$ Masculino & $19(47,5)$ \\
$\quad$ Feminino & $21(52,5)$ \\
Faixa etária, anos & \\
Até 20 & $3(7,5)$ \\
$21-30$ & $3(7,5)$ \\
$31-40$ & $1(2,5)$ \\
$41-50$ & $4(10,0)$ \\
$51-60$ & $4(10,0)$ \\
Acima de 60 & $25(62,5)$ \\
\hline
\end{tabular}

deles residiam na região intermediária de Maceió e 40\% $(\mathrm{n}=16)$ eram provenientes da região intermediária de Arapiraca, com maior concentração dos casos na capital do estado $(30 \% ; \mathrm{n}=12)$.

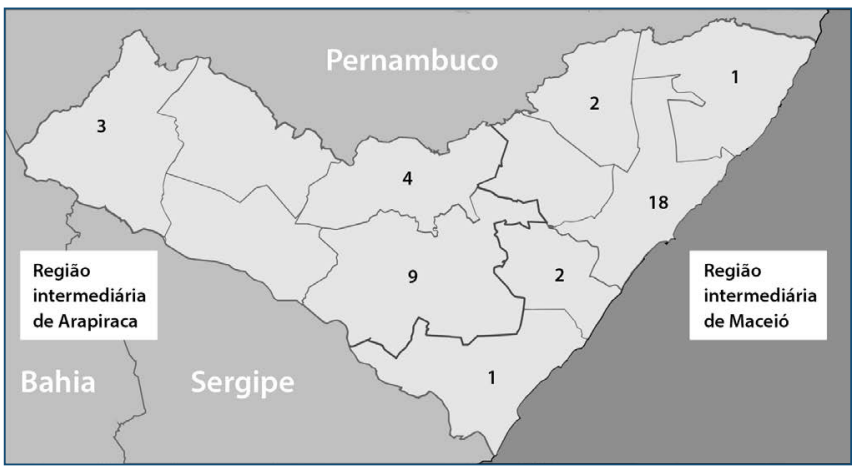

Figura 1. Distribuição geográfica dos transplantes de córnea por região intermediária e imediata em Alagoas, de 2018 a 2019. As linhas finas delimitam as regiões imediatas, e a linha mais espessa delimita as duas regiões intermediárias do estado.

Com relação às regiões imediatas, $45 \%(n=18)$ dos pacientes eram oriundos de Maceió, 22,5\%(n=9) de Arapiraca, $10 \%(\mathrm{n}=4)$ de Palmeira dos Índios, 7,5\% $(\mathrm{n}=3)$ de Delmiro Gouveia, 5\% ( $\mathrm{n}=2)$ de São Miguel dos Campos, $5 \%(\mathrm{n}=2)$ de Penedo, 2,5\% ( $\mathrm{n}=1)$ de União dos Palmares e $2,5 \%(\mathrm{n}=1)$ de Porto Calvo.

Em relação à distribuição temporal, 62,5\%(n=25) dos pacientes realizaram o transplante no ano de 2018 e 57,5\% $(n=15)$ em 2019. Os diagnósticos oculares encontrados foram ceratopatia bolhosa do pseudofácico $(27,5 \%$; $n=11)$, descemetocele $(22,5 \% ; n=9)$, falência tardia $(15 \% ; n=6)$, perfuração $(12,5 \% ; n=5)$, leucoma de profundidade não especificada $(7,5 \% ; n=3)$, ceratocone $(5 \% ; n=2)$, degeneração corneana ( $5 \% ; n=2)$, falência primária $(2,5 \% ; n=1)$ e distrofia de Fuchs $(2,5 \% ; n=1)$.

O tempo médio geral em lista de espera para a realização do transplante de córnea, desde o momento da inscrição no Sistema Nacional de Transplante até a realização do procedimento, foi de 332,3 dias (11 meses), enquanto metade dos pacientes aguardou até 469 dias ( 1 ano e 3 meses). O tempo de espera, em dias, para cirurgias eletivas ou de urgência foi de 486 dias ( 2 anos e 4 meses) e de 12,8 dias, respectivamente.

A tabela 2 apresenta o perfil clínico e cirúrgico dos 40 procedimentos de transplante de córnea realizados durante o período estudado.

Tabela 2. Perfil clínico-cirúrgico dos transplantes de córnea acompanhados no hospital universitário ( $n=40$ olhos, 40 pacientes), de 2018 a 2019

$\begin{array}{lc}\text { Características } & \mathbf{n}(\%) \\ \text { Falência do enxerto anterior } & 8(20,0) \\ \text { Sim } & 32(80,0) \\ \text { Não } & \\ \text { Olho operado } & 12(30,0) \\ \text { Direito } & 28(70,0) \\ \text { Esquerdo } & \\ \text { Propósito do transplante } & 26(65,0) \\ \text { Óptico } & 6(15,0) \\ \text { Tectônico } & 7(17,5) \\ \text { Terapêutico } & 1(2,5) \\ \text { Cosmético } & \\ \text { Urgência cirúrgica } & 27(67,5) \\ \text { Eletiva } & 13(32,5) \\ \text { Urgência } & \\ \text { Cirurgia combinada com extração de catarata } & 9(22,5) \\ \text { Sim } & 31(77,5) \\ \text { Não } & \\ \text { Complicações } & 2(5,0) \\ \text { Sim } & 38(95,0) \\ \text { Não } & \end{array}$

Do total de transplantes de córnea, 70\% $(\mathrm{n}=28)$ foram realizados no olho esquerdo, $65 \%(\mathrm{n}=26)$ com propósito óptico, 17,5\% (n=7) foram terapêuticos, 15\% ( $\mathrm{n}=6)$ foram tectônicos e 2,5\% $(n=1)$ foram cosméticos. A ceratoplastia penetrante era a única disponível no serviço em questão, tendo sido a técnica cirúrgica comum a todos os transplantes, e a associação de extração de catarata ocorreu em 22,5\% $(n=9)$ dos procedimentos. Foram submetidos ao retransplante $22,5 \%(n=9)$, sendo $88,9 \%$ destes $(n=8)$ por apresentarem falência do enxerto anterior e $11,1 \%(\mathrm{n}=1)$ por perfuração ocular do enxerto. Dois (5\%) transplantes apresentaram complicações peroperatórias, que consistiram em perda vítrea e expulsão espontânea do cristalino.

\section{DISCUSSÃO}

A descrição do perfil clínico dos transplantes penetrantes de córnea apresentada no presente estudo aponta discreta predominância de indivíduos do sexo feminino e distúrbios da córnea distribuídos por todas as faixas 
etárias, com maior predominância em pacientes acima de 60 anos.

As indicações para um transplante de córnea são distintas entre os países e dependem de condições socioeconômicas e demográficas de cada população, podendo variar, ainda, de acordo com o período de tempo estudado e os fatores ambientais e culturais. ${ }^{(7,8)}$ Elas refletem, assim, a mudança na incidência e no tratamento das doenças corneanas, bem como os efeitos de procedimentos cirúrgicos oculares. ${ }^{(8)} \mathrm{Na}$ literatura mundial, as indicações mais frequentes incluem ceratocone, ceratite infecciosa, cicatrizes corneanas e ceratopatia bolhosa. ${ }^{(7,8)}$ A principal condição indicadora para o transplante de córnea no serviço estudado foi a ceratopatia bolhosa, caracterizada pelo edema corneano estromal acompanhado de bolhas epiteliais e subepiteliais devido à perda de células e/ou à disfunção da função endotelial, corroborando com estudos realizados no Pará, ${ }^{(9)}$ em Sergipe ${ }^{(10)}$ e Tóquio. ${ }^{(7)} \mathrm{Com}$ a popularização das cirurgias de facoemulsificação nas últimas décadas e o aumento dos implantes de lente intraocular, houve aumento importante de casos de descompensação corneana e necessidade de realização de transplante de córnea. Já estudos conduzidos em outras partes do Brasil, América Latina e Europa revelam o ceratocone como principal indicação para ceratoplastia. ${ }^{(8,11)}$

A distribuição temporal, por sua vez, é marcada por expressiva diminuição do número de transplantes no ano de 2019, em comparação a 2018. Esse número decrescente de ceratoplastias se deve à inativação do Banco de Tecidos Oculares por alguns meses em 2019, o que levou à ausência de captação de córneas do estado nesse período, com dependência da colaboração de outros estados.

Além disso, a distribuição geográfica dos casos infere a existência de heterogeneidade em relação às regiões imediatas do estado, com maior prevalência na região imediata de Maceió, mais precisamente na capital do estado e na região metropolitana. Tal indicador reflete a própria densidade demográfica da região e também pode remeter à existência de problemas como dificuldades de acesso aos serviços especializados e consequente prejuízo para o diagnóstico precoce dos casos cujo transplante de córnea represente a conduta terapêutica adequada, consistindo no diagnóstico de um problema de saúde pública.

O tempo médio em fila de espera para o transplante de córnea foi de 486 dias ( 2 anos e 4 meses) para transplantes eletivos e 12,8 dias para urgências. No contexto nacional, o tempo de espera para a realização do transplante de córnea varia de acordo com cada região, observando espera de 1 ano e 3 meses no Pará(9) e média de 4 meses para os estados de São Paulo ${ }^{(12)}$ e Pernambuco, ${ }^{(13)}$ com tendência de homogeneização do tempo de espera em torno de 134 dias. ${ }^{(10,12)}$

No Brasil, a fila de transplantes de córnea é estadual e única, cujo atendimento ocorre por ordem de chegada, considerando critérios técnicos de priorização. ${ }^{(14)}$ As filas longas resultam do descompasso entre oferta e demanda, exercendo impacto negativo sobre bem-estar psicossocial, probabilidades de cura e sequelas ao paciente, aos familiares e à sociedade. ${ }^{(15)} \mathrm{O}$ tempo de espera prolongado para realização da cirurgia envolve vários fatores, sobretudo os relacionados ao sistema doação-captação, como queda no número de doadores efetivos e rastreamento e busca ativa de potenciais doadores ineficiente. O desenvolvimento de políticas internas e externas capazes de incentivar a doação, como o treinamento de profissionais de saúde e campanhas educativas para a população, faz-se necessário. ${ }^{(16)}$

A única técnica cirúrgica disponível no serviço era a ceratoplastia penetrante, que é o procedimento dominante há mais de meio século e atende com sucesso a maioria das causas de cegueira corneal. Entretanto, trata-se de técnica em que ocorre a substituição de todas as camadas da córnea, comportando maior risco de rejeição e complicações peroperatórias, pela descompressão do segmento anterior e pelo tempo cirúrgico a "céu aberto", pelo maior número de suturas com resultado óptico mais tardio, pelo maior risco de infecção, etc.

Os transplantes lamelares, anteriores ou posteriores, em que ocorre a substituição parcial de tecido corneano, atualmente são o padrão-ouro no tratamento de afecções que acometem apenas camadas específicas córnea. Possuem inúmeras vantagens em relação à ceratoplastia penetrante, incluindo a recuperação visual mais rápida e o menor risco de rejeição e de complicações peroperatórias. Apesar dos benefícios, eles não são realizados na instituição pela falta de profissionais habilitados, maior curva de aprendizado e pouca oferta de tecido ao banco de olhos.

Em relação à coleta de dados, por sua vez, por se tratar de uma pesquisa cuja fonte foi oriunda de dados secundários, deve-se presumir que este estudo, assim como os demais que optem por essa técnica, apresenta alguns vieses que podem ser fatores limitantes, como as perdas de informações e as fragilidades dos registros e sistemas de informações.

\section{CONCLUSÃO}

A média de idade no momento da realização do transplante de córnea foi de 59 anos, e o paciente, em geral, era procedente da região intermediária de Maceió. A 
principal condição indicadora para o transplante de córnea foi a ceratopatia bolhosa, e o principal propósito foi óptico. A técnica cirúrgica utilizada em todos os casos foi a ceratoplastia penetrante, e o tempo médio de espera encontrado foi de, aproximadamente, 486 dias, para transplantes de córnea eletivos, e de 12,8 dias, para os casos de urgência.

O conhecimento do perfil epidemiológico dos transplantes de córnea em determinada região pode permitir a identificação de grupos de risco para fins de prevenção e implementação de cuidados que resultem em prognósticos mais favoráveis, bem como incentivar a implementação de políticas internas e externas para melhoria do sistema captação-doação.

\section{REFERÊNCIAS}

1. Pascolini D, Mariotti SP. Global estimates of visual impairment: 2010 . Br J Ophthalmol. 2012;96(5):614-8.

2. Foster A, Gilbert C, Johnson G. Changing patterns in global blindness: 1988-2008. Community Eye Health. 2008;21(67):37-9.

3. Chaurasia SS, Lim RR, Lakshminarayanan R, Mohan RR. Nanomedicine approaches for corneal diseases. J Funct Biomater. 2015;6(2):277-98.

4. Xu SC, Chow J, Liu J, Li L, Maslin JS, Chadha N, et al. Risk factors for visual impairment associated with corneal diseases in southern China. Clin Ophthalmol. 2016;10:777-82.

5. Papadopoulos K, Papakonstantinou D, Montgomery A, Solomou A. Social support and depression of adults with visual impairments. Res Dev Disabil. 2014;35(7):1734-41
6. Ple-Plakon PA, Shtein RM. Trends in corneal transplantation: indications and techniques. Curr Opin Ophthalmol. 2014;25(4):300-5.

7. Inoue K, Amano S, Oshika T, Sawa M, Tsuru T. A 10-year review of penetrating keratoplasty. Jpn J Ophthalmol. 2000;44(2):139-45.

8. Ting DS, Sau CY, Srinivasan S, Ramaesh K, Mantry S, Roberts F. Changing trends in keratoplasty in the West of Scotland: a 10-year review. $\mathrm{Br} \mathrm{J}$ Ophthalmol. 2012;96(3):405-8.

9. Sobrinho EF, Negrão BC, Almeida HG. [Epidemiological profile of patients waiting for penetrating keratoplasty in state of Pará, Brazil]. Rev Bras Oftalmol. 2011;70(6):384-90. Portuguese.

10. Araújo AA, Melo GB, Silva RL, Araújo Neta VM. Perfil epidemiológico dos pacientes na lista de espera para transplante de córnea no Estado de Sergipe. Arq Bras Oftalmol. 2004;67(4):613-6.

11. Tan JC, Holland SP, Dubord PJ, Moloney G, McCarthy M, Yeung SN. Evolving indications for and trends in keratoplasty in British Columbia, Canada, from 2002 to 2011: a 10-year review. Cornea. 2014;33(3):252-6.

12. Oliveira LA, Correa BS, Almeida Júnior GC, Ferrari MT, Kashiwabuchi LK. Influence of a single regional list for corneal transplantation on the eye bank of a university hospital. Arq Bras Oftalmol. 2003;66(5):631-5.

13. Almeida HG, Souza AC. Epidemiological profile of patients waiting for penetrating keratoplasty in state of Pernambuco - Brazil. Rev Bras Oftalmol. 2014;73(1):28-32.

14. Brasil. Ministério da Saúde. Portaria No 2.600, de 21 de outubro de 2009. Aprova o Regulamento Técnico do Sistema Nacional de Transplantes. Brasília, Diário Oficial da União; 2009 [citado 2021 Mar 16]. Disponível em: http://bvsms.saude.gov.br/bvs/saudelegis/gm/2009/ prt2600_21_10_2009.html

15. Marinho A. Um estudo sobre as filas para transplantes no Sistema Único de Saúde brasileiro. Cad Saúde Pública (Rio de Janeiro). 2006;22(10):222939.

16. Farias RJ, Sousa LB. Impacto do marketing dos processos de divulgação nas doações de córneas a um banco de tecidos oculares humanos e avaliação do perfil socioeconômico de seus doadores. Arq Bras Oftalmol. 2008;71(1):28-33. 